\title{
Novel Lipid Species for Detecting and Predicting Atrial Fibrillation in Patients With Type 2 Diabetes
}

\author{
Yow Keat Tham, ${ }^{1,2}$ Kaushala S. Jayawardana, ${ }^{1}$ Zahir H. Alshehry, ${ }^{1,3}$ Corey Giles, ${ }^{1}$ Kevin Huynh, ${ }^{1}$ \\ Adam Alexander T. Smith, ${ }^{1}$ Jenny Y.Y. Ooi, ${ }^{1,2}$ Sophia Zoungas, ${ }^{4,5}$ Graham S. Hillis, ${ }^{4,6}$ John Chalmers, ${ }^{4}$ \\ Peter J. Meikle,1,2,3 and Julie R. McMullen'1,2,7,8 \\ Diabetes 2021;70:255-261 | https://doi.org/10.2337/db20-0653
}

\begin{abstract}
The incidence of atrial fibrillation (AF) is higher in patients with diabetes. The goal of this study was to assess if the addition of plasma lipids to traditional risk factors could improve the ability to detect and predict future AF in patients with type 2 diabetes. Logistic regression models were used to identify lipids associated with AF or future AF from plasma lipids $(n=316)$ measured from participants in the ADVANCE trial $(n=3,772)$. To gain mechanistic insight, follow-up lipid analysis was undertaken in a mouse model that has an insulin-resistant heart and is susceptible to AF. Sphingolipids, cholesteryl esters, and phospholipids were associated with AF prevalence, whereas two monosialodihexosylganglioside (GM3) ganglioside species were associated with future AF. For AF detection and prediction, addition of six and three lipids, respectively, to a base model ( $n=12$ conventional risk factors) increased the $\mathrm{C}$-statistics (detection: from 0.661 to 0.725 ; prediction: from 0.674 to 0.715 ) and categorical net reclassification indices. The GM3(d18:1/24:1) level was lower in patients in whom AF developed, improved the C-statistic for the prediction of future AF, and was lower in the plasma of the mouse model susceptible to AF. This study demonstrates that plasma lipids have the potential to improve the detection and prediction of AF in patients with diabetes.
\end{abstract}

Atrial fibrillation (AF) is the most common rhythm disorder of the heart, affecting $1-4 \%$ of the general population $(1,2)$, but $\sim 15 \%$ of patients with diabetes $(2-4)$. It is associated with considerable morbidity, increased mortality, and a major burden on health care resources. When AF is permanent, the pathology is typically well advanced and challenging to treat. There is an urgent need for 1) early detection of AF with strategies that are resource efficient, accessible, and scalable; and 2) strategies to predict patients at risk for AF so preventive strategies can be implemented (2).

Previous studies have highlighted the potential of proteins, genes, metabolites, and lipids to serve as potential biomarkers for $\mathrm{AF}$ (5). An advantage of metabolites and lipids is that they represent direct signatures of biochemical activity and a functional readout of a phenotype or disease state, capturing genetic and environmental influences $(6,7)$. For this study, our goal was to profile the plasma lipidome ( $>300$ lipids) from a cohort of patients with diabetes $(n=3,772$; a subcohort of the Action in Diabetes and Vascular Disease: Preterax and DiamicronMR Controlled Evaluation [ADVANCE] trial) to ascertain if lipids are associated with $\mathrm{AF}$ and improve the ability to predict future AF. To gain mechanistic insight, follow-up

\footnotetext{
${ }^{1}$ Baker Heart and Diabetes Institute, Melbourne, Victoria, Australia

2Department of Diabetes, Central Clinical School, Monash University, Melbourne, Victoria, Australia

${ }^{3}$ Department of Biochemistry and Molecular Biology, University of Melbourne, Parkville, Victoria, Australia

${ }^{4}$ The George Institute for Global Health, University of New South Wales, Sydney, New South Wales, Australia

${ }^{5}$ School of Public Health and Preventive Medicine, Monash University, Melbourne, Victoria, Australia

${ }^{6}$ Royal Perth Hospital/University of Western Australia, Perth, Western Australia, Australia

${ }^{7}$ Department of Physiology, Monash University, Clayton, Victoria, Australia

${ }^{8}$ Department of Physiology, Anatomy and Microbiology, La Trobe University, Bundoora, Victoria, Australia
}

Corresponding author: Julie R. McMullen, julie.mcmullen@baker.edu.au, or Peter J. Meikle, peter.meikle@baker.edu.au

Received 22 June 2020 and accepted 15 0ctober 2020

This article contains supplementary material online at https://doi.org/10.2337/ figshare.13102979.

P.J.M. and J.R.M. are joint senior authors.

Z.H.A. is currently affiliated with King Fahad Medical City, Riyadh, Saudi Arabia. (C) 2020 by the American Diabetes Association. Readers may use this article as long as the work is properly cited, the use is educational and not for profit, and the work is not altered. More information is available at https://www.diabetesjournals .org/content/license. 
lipid analysis was undertaken in a mouse model that had an insulin-resistant heart and was susceptible to AF.

\section{RESEARCH DESIGN AND METHODS}

\section{Study Approval}

The ADVANCE trial was approved by the University of Sydney Human Research Ethics Committee (Sydney, New South Wales, Australia). Analysis of archived plasma samples was approved by the Alfred Hospital Ethics Committee (Melbourne, Victoria, Australia). Animal care and experimentation were approved by the Alfred Research Alliance Animal Ethics Committee (Melbourne, Victoria, Australia). Prior to euthanasia, blood was collected and processed as described (8).

\section{Study Populations}

The ADVANCE trial ( $n=11,140$ participants with type 2 diabetes) was a randomized factorial trial (double-blind comparison of blood pressure lowering vs. placebo, and open comparison of intensive vs. standard glucose control [9]). A previously selected cohort of case patients with cardiovascular disease $(n=3,779)$ was used for these analyses $(9,10)$. We conducted an observational cohort study within the trial on plasma samples collected at baseline (before assignment to a treatment arm) (Fig. 1). The treatment arms had no effect on AF (11).

\section{Subset 1 (AF Baseline)}

The cohort for AF detection included 3,772 participants (those without $\mathrm{AF}$ [hereafter, non-AF]: $n=3,368$; AF: $n=$ 404). AF was assessed by 12 -lead electrocardiogram (ECG) by combining two variables: current $\mathrm{AF}$ at the baseline visit or previous $\mathrm{AF}$ within 3 months of the baseline visit.

\section{Subset 2 (AF Future)}

The cohort for the prediction of future AF included 2,238 participants (non-AF: $n=2,054$; future AF: $n=184$ ). A patient was classified as having future $\mathrm{AF}$ if there was no evidence of $\mathrm{AF}$ at baseline or within the past 3 months, and AF was detected by ECG at the 24-, 48-, or 60-month study visit. Patients without follow-up ECG data $(n=1,534)$ were excluded. Patient characteristics of non-AF groups in cohorts 1 and 2 were comparable, except for a mean age difference of 1 year (Supplementary Tables 1-3).

\section{Heart-Specific Mouse Model With Reduced Phosphoinositide 3-Kinase (PI3K) and an Insulin-Resistant Heart}

Lipid profiling was performed on plasma from adult (1013 weeks old) male and female, heart-specific, dominant negative PI3K transgenic mice (dnPI3K-reduced cardiac PI3K activity of $\sim 77 \%$ [12]) and nontransgenic control mice (Fig. 1).

\section{Lipidomic Profiling}

Lipids were extracted from $10 \mu \mathrm{L}$ of human plasma and abundances measured in our previous study; information regarding batch effects and inter- and intra-assay coefficients of variation are described (10). Lipids were extracted from $10 \mu \mathrm{L}$ of mouse plasma and analyzed as described $(8,13)$.

\section{Statistics}

Mann-Whitney $U$ test and $\chi^{2}$ tests were used for continuous and categorical variables, respectively. Lipid data were log10-transformed and scaled by SD. Logistic regression models that were adjusted for 12 covariates (Supplementary Table 1; clinical lipids, covariates selected by the CHARGE-AF Consortium [14]) were used to determine the association of individual lipids with the prevalence and prediction of AF. Logistic and linear regression $P$ values were corrected for multiple comparisons using the Benjamini-Hochberg method $(P<0.05)$. Cluster analysis was performed in R. Ranking of lipids was performed using Akaike information criterionbased forward selection of lipids into multivariate models (10) and assessed using the C-statistics for 5-year risk, continuous and categorical net reclassification index (NRI), integrated discrimination indexes (IDI) and relative IDI with fivefold cross-validation ( $n=200$ repeats). Categorical NRI was calculated on the basis of 5 -year risk categories of $<2.5 \%$, $2.5-5 \%$, and $>5 \%$.

\section{Data and Resource Availability}

All data generated and analyzed during this study are included here and in the Supplementary Material. No applicable resources were generated or analyzed during this study.

\section{RESULTS}

\section{Baseline Characteristics}

Characteristics of patients in subset 1 (AF Baseline) ( $n=$ 3,368 participants in the non-AF group and $n=404$ in the AF group at baseline) (Fig. 1) are provided in Supplementary Table 1. Older age and obesity are risk factors for AF, and patients with $\mathrm{AF}$ were significantly older and heavier.

Subset 2 (AF Future) ( $n=2,238$ participants) represented participants from subset 1 who did not have AF at baseline and for whom an ECG was performed at a follow-up time point (24, 48, and/or 60 months). AF was subsequently identified in 184 patients (Supplementary Table 2).

\section{Lipids Associated With Baseline and Future AF}

Logistic regression analysis was conducted on data from subsets 1 and 2 (Supplementary Tables 4 and 5) after adjusting for 12 covariates (Supplementary Table 1). For subset 1 (AF Baseline), 42 lipids ( $n=14$ sphingolipids, 10 neutral lipids, 6 lysophospholipids, and 12 phospholipids) were associated with the presence of $\mathrm{AF}(n=8$ negative; $n=34$ positive) (Fig. 2A). Although univariate analysis showed that total cholesterol was associated with prevalent AF (Supplementary Table 1), because clinical lipids were included in the multivariate model, the 42 lipids identified were independent of clinical lipids (Supplementary Table 6). All six dihexosylceramide species were positively associated with the prevalence of AF (Fig. 2A). 


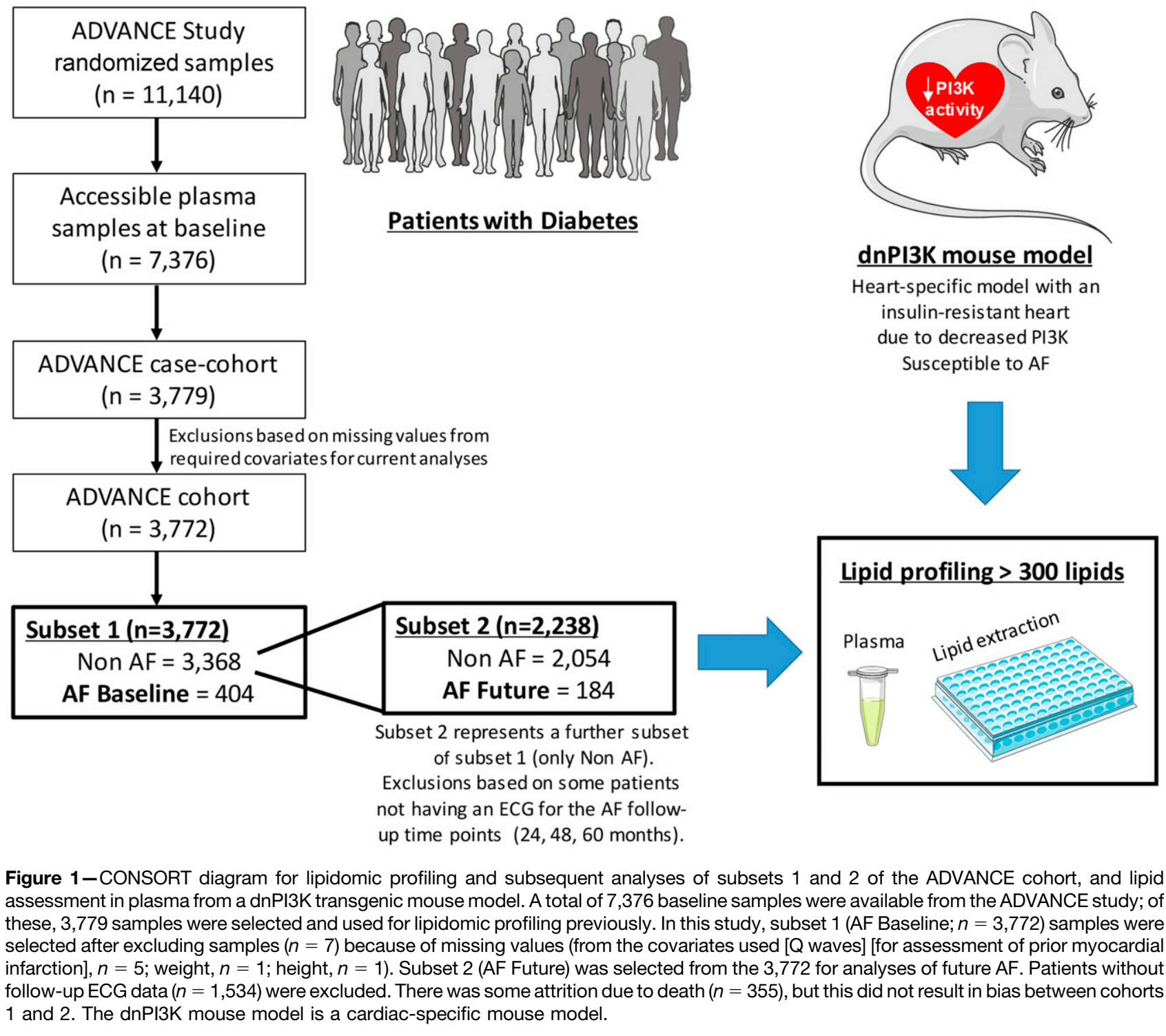

In subset 2 (AF Future), two species of monosialodihexosylganglioside (GM3) ganglioside were negatively associated with future AF (Fig. 2B). Given the positive association of dihexosylceramide (Hex2Cer) species with $\mathrm{AF}$ in subset 1 (Fig. $2 A$ ) and the inverse association of GM3 species in subset 2 (Fig. $2 B$ ), we evaluated the ratio of Hex2Cer to GM3 because Hex2Cer is a precursor substrate for GM3 (Supplementary Fig. 1A). Five of six Hex2Cer-to-GM3 ratios were significantly associated with $\mathrm{AF}$ in subset 1 (Supplementary Fig. 1B), whereas two of the six Hex2Cer-to-GM3 ratios were associated with the development of future AF in subset 2 (Supplementary Fig. $1 C$ ).

Cluster analysis (Supplementary Fig. 2) identified other species from the same class, which correlated with individual lipids associated with $\mathrm{AF}$ at baseline (Fig. 2A). By contrast, only GM3 species were correlated for the prediction of future AF (Supplementary Fig. 2).

\section{Multivariate Models Identified Lipids That Improved on Traditional Risk Factors for the Detection and Prediction of AF}

Sequential addition of ranked lipids to the base model led to an increase in the $\mathrm{C}$-statistic for both the detection of $\mathrm{AF}$ at baseline and prediction of future $\mathrm{AF}$ (Fig. $2 C$ and $D$ ). There was an inflection point after the addition to the model of six lipids (AF detection) and three lipids (future AF). To prevent overfitting, we limited the models to 12 covariates and six and three lipids for the baseline AF and future AF models, respectively. This improved the $\mathrm{C}$-statistics, categorical and continuous NRI, IDIs, and relative IDIs (Table 1).

\section{Depressed Plasma GM3 Lipids in a Heart-Specific Mouse Model With Reduced PI3K and an Insulin-Resistant Heart}

To examine whether the insulin-resistant heart in a setting of diabetes has the potential to contribute to the lipid 
A

\section{Detecting AF}

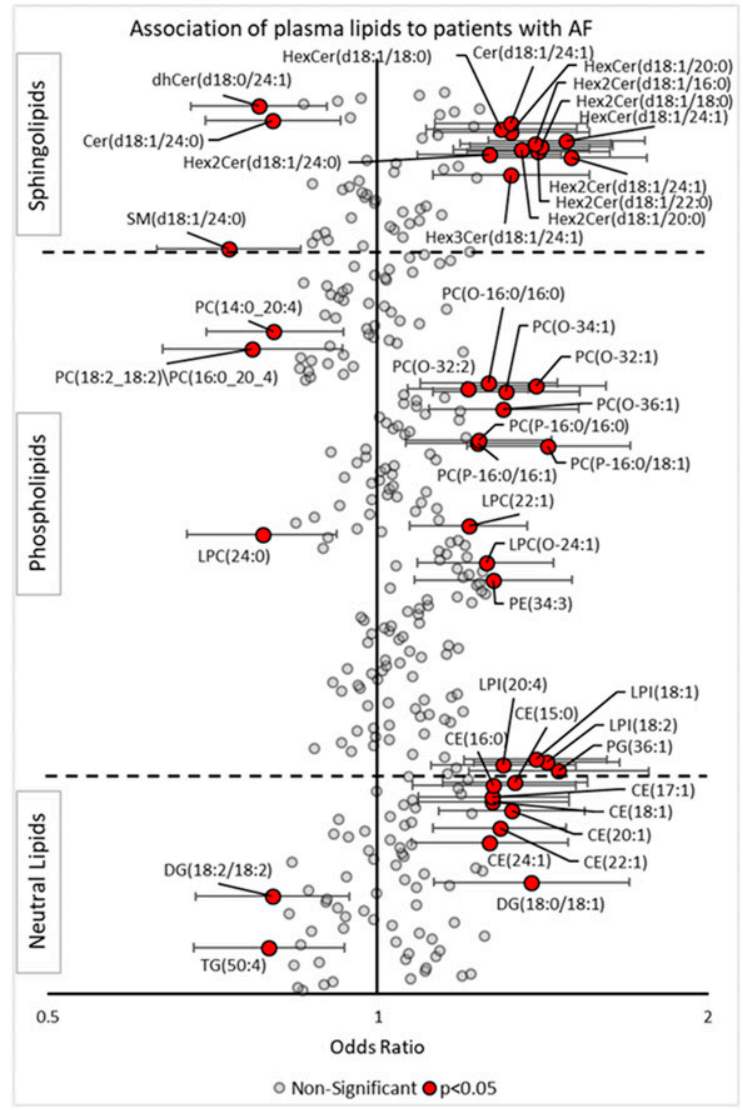

C

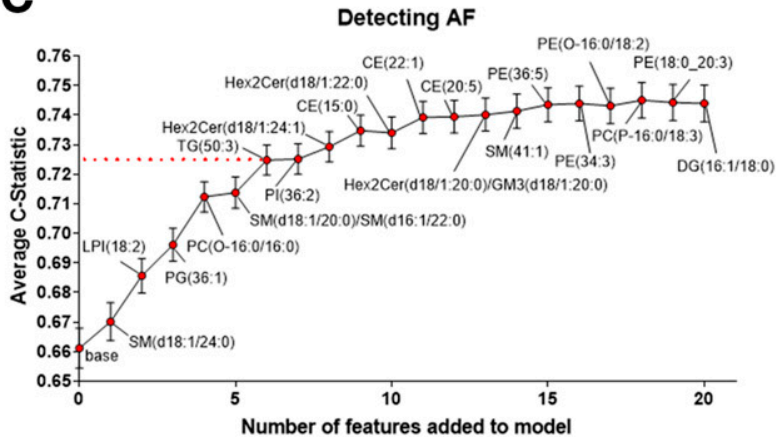

B

\section{Predicting AF}

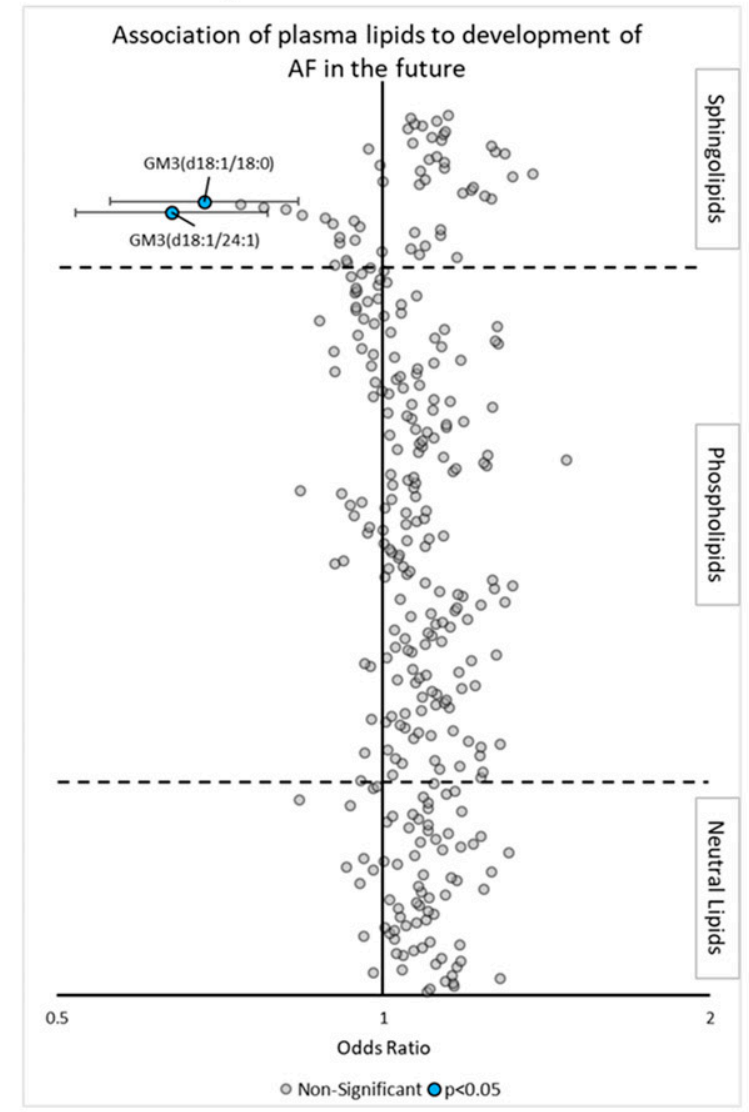

D

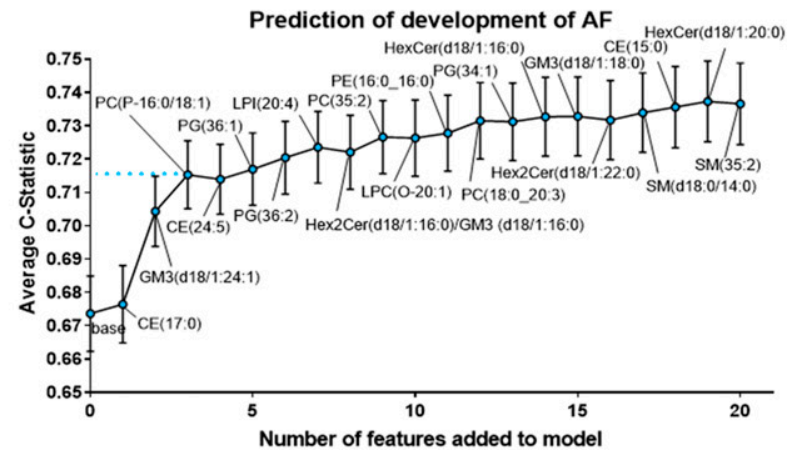

Figure 2-Association and predictive modeling of plasma lipids in detecting AF in ADVANCE subset $1(n=3,772)(A$ and $C)$, and patients in whom AF developed in the future (ADVANCE subset 2; $n=2,238)(B$ and $D$ ). $A$ and $B$ : Logistic regression model of individual lipid species against prevalence of AF at baseline (subset 1, AF Baseline) and future AF (subset 2, AF Future) adjusting for 12 covariates (namely, age, weight, height, systolic blood pressure, diastolic blood pressure, total cholesterol, HDL cholesterol, triglycerides, myocardial infarction, antihypertensive medication used, current smoking status, and hospital admission for heart failure). Odds ratios and $95 \%$ Cls are shown. Lipid levels in red $(A)$ and blue $(B)$ were significant $(P<0.05)$ after applying the Benjamini-Hochberg correction. $C$ and $D$ : Logistic regression models used to determine improvement in the $\mathrm{C}$-statistic to detect or predict the incidence of AF by adding lipids to the base model, consisting of the 12 covariates. Logistic regression models were developed using forward stepwise feature selection by Akaike information criterion reduction. Analysis was conducted in a fivefold crossvalidated framework (repeated 200 times). Ranked lipids are shown sequentially as features added to the base model. CE, cholesteryl ester; Cer, ceramide; DG, diacylglycerol; dhCer, dihydroceramide; GM3, monosialodihexosylganglioside; HexCer, monohexosylceramide; Hex2Cer, dihexosylceramide; Hex3Cer, trihexosylceramide; LPC, Iysophosphatidylcholine; LPC(O), lysoalkylphosphatidylcholine; LPI, lysophosphatidylinositol; PC, phosphatidylcholine; $\mathrm{PC}(\mathrm{O})$, alkylphosphatidylcholine; $\mathrm{PC}(\mathrm{P})$, alkenylphosphatidylcholine; $\mathrm{PE}$, phosphatidylethanolamine; $\mathrm{PE}(\mathrm{O})$, alkylphosphatidylethanolamine; PG, phosphatidylglycerol; PI, phosphatidylinositol; SM, sphingomyelin; TG, triacylglycerol. 


\begin{tabular}{|c|c|c|c|c|c|}
\hline Feature & C-Statistic & Continuous NRI & Categorical NRI & IDI & Relative IDI \\
\hline \multicolumn{6}{|c|}{ Detecting incidence of AF } \\
\hline Base model ${ }^{\star}$ & $\begin{array}{c}0.661 \\
(0.653-0.668)\end{array}$ & Reference & Reference & Reference & Reference \\
\hline $\begin{array}{l}\text { Base model }+ \text { six } \\
\text { lipid species\# }\end{array}$ & $\begin{array}{c}0.725 \\
(0.720-0.730)\end{array}$ & $\begin{array}{c}0.477 \\
(0.450-0.504)\end{array}$ & $\begin{array}{c}0.124 \\
(0.108-0.141)\end{array}$ & $\begin{array}{c}0.044 \\
(0.043-0.046)\end{array}$ & $\begin{array}{c}0.881 \\
(0.839-0.922)\end{array}$ \\
\hline \multicolumn{6}{|c|}{ Predicting incidence of $\mathrm{AF}$} \\
\hline Base model ${ }^{*}$ & $\begin{array}{c}0.674 \\
(0.662-0.685)\end{array}$ & Reference & Reference & Reference & Reference \\
\hline $\begin{array}{l}\text { Base model + three } \\
\text { lipid species } t\end{array}$ & $\begin{array}{c}0.715 \\
(0.705-0.725)\end{array}$ & $\begin{array}{c}0.434 \\
(0.372-0.495)\end{array}$ & $\begin{array}{c}0.084 \\
(0.049-0.119)\end{array}$ & $\begin{array}{c}0.017 \\
(0.015-0.019)\end{array}$ & $\begin{array}{c}0.439 \\
(0.386-0.492)\end{array}$ \\
\hline \multicolumn{6}{|c|}{$\begin{array}{l}\text { Data are reported as No. }(95 \% \mathrm{Cl}) \text { unless otherwise indicated. CE, cholesteryl ester; DG, diacylglycerol; GM3, monosialodihexosyl- } \\
\text { ganglioside; HexCer, monohexosylceramide; LPC(O), lysoalkylphosphatidylcholine; PC(O), alkylphosphatidylcholine; PC(P), alkenyl- } \\
\text { phosphatidylcholine; PE(O), alkylphosphatidylethanolamine. *The base model consisted of the following } 12 \text { covariates: age, weight, } \\
\text { height, systolic blood pressure, diastolic blood pressure, total cholesterol, HDL cholesterol, triglycerides, myocardial infarction, } \\
\text { antihypertensive medication used, current smoking status, and hospital admission for heart failure. \#Ranked lipids for the detection of AF } \\
\text { were SM(d18:1/24:0), LPI(18:2), PG(36:1), PC(O-16:0/16:0), SM(d18:1/20:0)/SM(d16:1/22:0), and TG(50:3). †Ranked lipids for the } \\
\text { prediction of AF incidence were CE(17:0), GM3(d18:1/24:1), and PC(P-16:0/18:1). }\end{array}$} \\
\hline
\end{tabular}

profile in the plasma, we undertook lipid profiling in a cardiac-specific dnPI3K transgenic mouse model. The mouse model is characterized as a heart-specific model of type 2 diabetes because it has defective cardiac insulin signaling due to depressed PI3K and develops severe cardiomyopathy and increased susceptibility to AF in response to cardiac stress (15-19). GM3, sphingomyelin (SM), phosphatidylserine, and oxidized species classes were decreased in plasma from dnPI3K mice (Supplementary Fig. 3). Here, we focused on levels of GM3 lipids, which were lower and clustered together in patients in whom AF subsequently developed (Figs. $2 B$ and $3 A$ and $B$ and Supplementary Fig. 2). Levels of three of five GM3 lipids were lower in plasma from dnPI3K mice (Fig. 3C).

\section{DISCUSSION}

New strategies and biomarkers are urgently required to identify those at risk for $\mathrm{AF}$, but these strategies and biomarkers are likely to differ on the basis of underlying conditions, including diabetes. The key findings of this study were: 1) 42 lipids were associated with the presence of $\mathrm{AF}$, and the addition of six lipids improved the accuracy of a base model ( $n=12$ covariates and conventional risk factors for $\mathrm{AF}$ ), and 2) two lipids were associated with the development of future AF, and the inclusion of three lipids improved the ability to predict future AF development. Finally, by assessing plasma lipids in a mouse model with an insulin-resistant heart, we gained insight as to how insulin resistance may contribute to altered plasma lipids.

Diabetes increases the risk for AF (3). Approximately $16 \%$ of patients in this study ( $n=588$ of 3,772 patients) either had AF at baseline or AF developed in these patients over the following 60 months. To our knowledge, this is the first study to undertake comprehensive lipidomic profiling ( $n>300$ lipids) in a large cohort of patients with diabetes screened for AF. Previous studies focused on smaller subsets of lipids, conventional lipids (e.g., HDL,
LDL, triacylglycerides), or much smaller cohorts of patients with AF (5,20-24).

\section{Lipids Associated With AF}

Of the 42 lipids associated with $\mathrm{AF}$, the two largest groups of lipids were cholesteryl esters and dihexosylceramides (Fig. 2A). Dihexosylceramides are a derivative of ceramides and serve as a precursor to GM3 gangliosides. Increased levels of circulating dihexosylceramides in the absence of an increase in GM3 gangliosides may indicate dysregulation within the sphingolipid pathway converting dihexosylceramide to GM3 ganglioside in patients with $\mathrm{AF}$ (Supplementary Fig. 1A).

Previous plasma lipidomic studies identified associations of specific lysophosphatidylcholine (LPC), LPE, phosphatidylcholine $(P C)$, phosphatidylethanolamine (PE), phosphatidylinositol and alkenylphosphatidylethanolamine species with $\mathrm{AF}(20,21)$. Although we did not observe associations with the same specific species, there were associations in lipids from the same lipid classes (i.e., LPC, PC, and PE) (Fig. 2A). A major difference between our cohort and previous work was that the ADVANCE cohort consisted entirely of patients with type 2 diabetes.

In our previous lipidomics study from the ADVANCE trial, for the prediction of cardiovascular events, the addition of seven lipids to the base model increased the $\mathrm{C}$-statistic from 0.680 to 0.700 (10). Using a similar approach here, the addition of six lipids [SM(d18:1/24:0), lysophosphatidylinositol (LPI)(18:2), phosphatidylglycerol (PG)(36:1), PC(O-16:0/ 16:0), SM(d18:1/20:0)/SM(d16:1/22:0), and triacylglycerol (TG)(50:3)] improved the C-statistic even more (from 0.661 to 0.725). Three of the six lipids were also associated with $\mathrm{AF}$ at baseline $[\mathrm{SM}(\mathrm{d} 18: 1 / 24: 0)$ level decreased; LPI(18:2) and PC(O-16:0/16:0) levels increased] (Fig. 2A). We previously identified a decrease in plasma $\mathrm{SM}(\mathrm{d} 18: 1 / 24: 0)$ levels in a mouse model with substantial cardiac pathology due to severe pressure overload (8). These mice had enlarged atria, which predispose the heart to AF. 

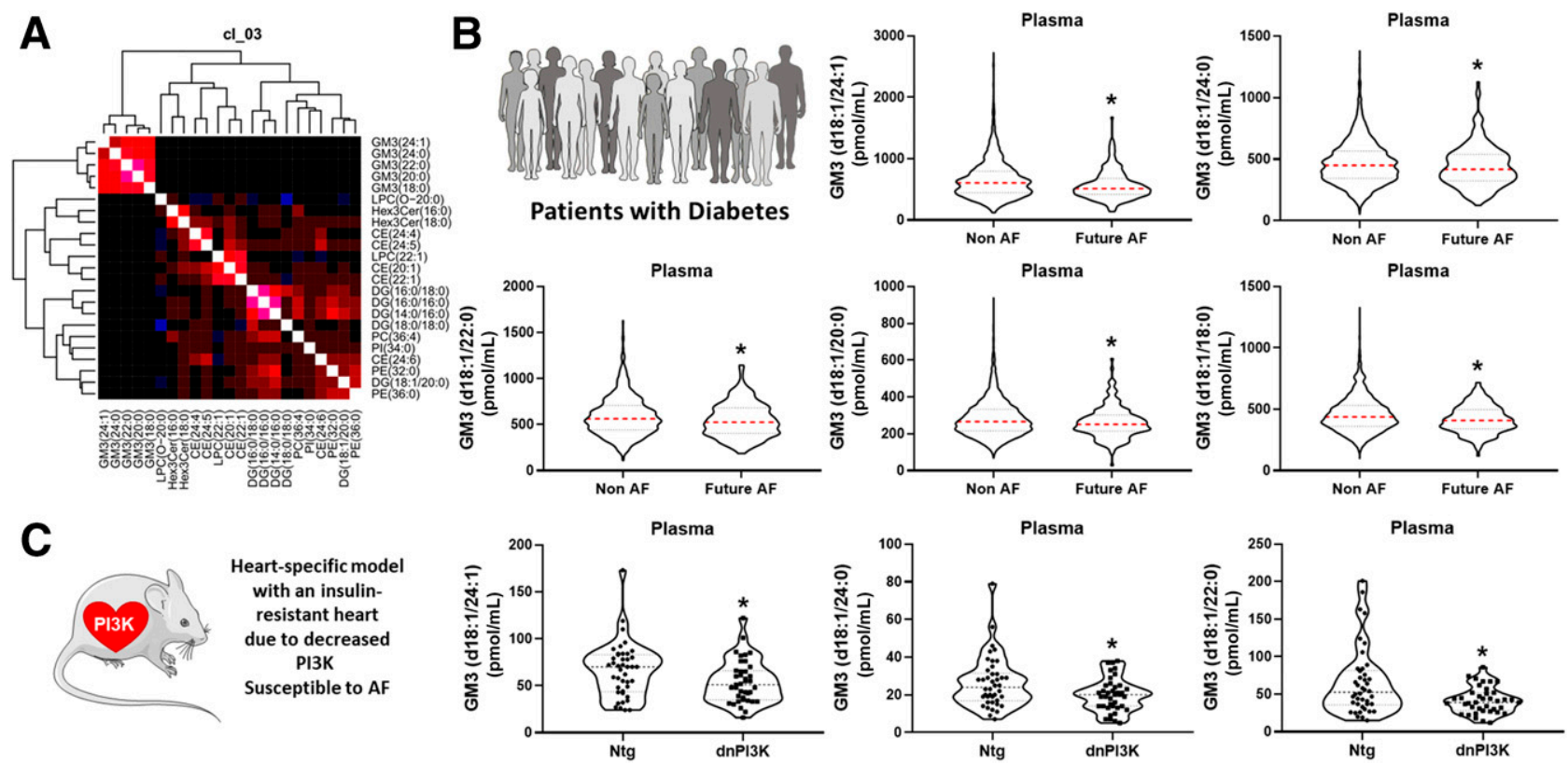

Figure 3-GM3 lipid species in plasma from patients in subset 2 (AF Future) and dnPI3K mice. A: Lipids clustered with GM3 lipids from Fig. $2 B$ (cluster 3 from complete heat map presented in Supplementary Fig. 2). Color scale: cyan (correlation [corr] $=-1$ ) to blue to black $(\operatorname{corr}=0$ ) to red to magenta (corr $=1$ ); diagonal (self corr $=1$ ) shown in white. $B$ and $C$ : GM3 lipid levels that were significantly changed in ADVANCE subset 2 and dnPI3K mice. ${ }^{*} P<0.05$ vs. non-AF. For mouse lipidomic data, the Shapiro-Wilk test was used to check for normality before additional testing. ${ }^{\star} P<0.05$ vs. nontransgenic (Ntg) via Mann-Whitney test. Non-AF, $n=2,054$; AF Future, $n=184$. Animal numbers: Ntg, $n=42$ ( $n=25$ male; $n=17$ female); dnPI3K, $n=39$ ( $n=21$ male; $n=18$ female).

Lipids Associated With the Future Development of AF

The ability to better identify people at risk for $\mathrm{AF}$, and provide interventions to prevent $\mathrm{AF}$, would have enormous health and economic benefits. Two GM3 lipids were inversely associated with the development of $\mathrm{AF}$ at a later time (Fig. $2 B$ ). The addition of three lipids improved the ability to predict future AF (C-statistic: from 0.674 to 0.715); GM3(d18:1/24:1) increased the C-statistic the most. In settings of type 2 diabetes and obesity, GM3 gangliosides have been implicated mechanistically via a disruption in insulin signaling inducing insulin resistance (25). Numerous studies have identified associations between lipids and various diseases. Mechanistic insight into how these lipids reflect and/or contribute to pathology are important to assess which lipids have the most potential as biomarkers or drug targets. In our study, levels of several GM3 lipids were decreased in patients in whom AF subsequently developed (Fig. $3 A$ and $B$ ), and GM3 lipid levels also decreased in the plasma of dnPI3K mice, which have an insulin-resistant heart and are susceptible to AF in cardiac-stress settings (Fig. 3C). Collectively, these data provide a potential mechanism whereby the insulinresistant diabetic heart might contribute to the differential levels of lipids in the circulation.

\section{Limitations and Future Directions}

It will be important to validate our lipid markers and modeling in independent cohorts of patients with diabetes. AF can be missed in patients with paroxysmal or intermittent AF. However, any misclassification of patients in our cohort has been estimated to represent $\sim 3 \%$ of the group (11) and will not change the associations identified. From mechanistic and therapeutic perspectives, it will be important to assess whether reduced circulating levels of GM3 contribute to the development of AF or are a consequence of dysregulated cardiac metabolism due to cardiac insulin resistance.

In summary, we identified circulating lipids that were associated with the presence of AF and the development of future $\mathrm{AF}$ in patients with type 2 diabetes. We have also shown that lipids can improve the detection and prediction of AF over and above traditional risk factors in this setting. Incorporation of lipids into risk assessment could have a significant impact on improving patient outcomes.

Acknowledgments. The authors thank the investigators of the Action in Diabetes and Vascular Disease: Preterax and Diamicron-MR Controlled Evaluation (ADVANCE) trial and the patients who participated in these studies. The authors also thank Lydia Lim for assistance genotyping transgenic mice, and Natalie Mellett for assistance with lipidomic methods (both from Baker Heart and Diabetes Institute).

Funding. This study was funded by National Health and Medical Research Council project grants to J.R.M. $(1045585,1125514)$ and in part by the Victorian Government's Operational Infrastructure Support Program. P.J.M. and J.R.M. are National Health and Research Council Senior Research Fellows (identifiers 1042095 and 1078985 , respectively). The ADVANCE study was funded by the National Health and Medical Research Council of Australia (grants 211086 and 358395, trial registration: https://clinicaltrials.gov. Clinical trial reg. no. NCT00145925). Z.H.A. was supported by a scholarship from King Fahad Medical City (Riyadh, Saudi Arabia). 
Duality of Interest. No potential conflicts of interest relevant to this article were reported.

Author Contributions. J.R.M. conceived the study. J.R.M. and P.J.M. designed the study. K.S.J. developed the statistical analysis protocols with input from P.J.M. The ADVANCE trial was conducted by S.Z., G.S.H., and J.C. The lipidomic analysis and data processing of the ADVANCE samples were performed previously by Z.H.A. C.G. and K.H. contributed to lipidomic methodology. C.G., K.H., and A.A.T.S. assisted with statistical analyses. Y.K.T. and J.Y.Y.O. performed mouse experiments. Y.K.T. performed lipidomic experiments, and analyses of mouse samples and lipid results from the ADVANCE trial in relation to atrial fibrillation. Y.K.T. and J.R.M. wrote the manuscript. All authors critically edited the manuscript. P.J.M. and J.R.M. are the guarantors of this work and, as such, had full access to all the data in the study and take responsibility for the integrity of the data and the accuracy of the data analysis.

\section{References}

1. Fuster V, Rydén LE, Cannom DS, et al.; American College of Cardiology/ American Heart Association Task Force on Practice Guidelines; European Society of Cardiology Committee for Practice Guidelines; European Heart Rhythm Association; Heart Rhythm Society. ACC/AHA/ESC 2006 guidelines for the management of patients with atrial fibrillation: a report of the American College of Cardiology/ American Heart Association Task Force on Practice Guidelines and the European Society of Cardiology Committee for Practice Guidelines (Writing Committee to Revise the 2001 Guidelines for the Management of Patients With Atrial Fibrillation): developed in collaboration with the European Heart Rhythm Association and the Heart Rhythm Society. Circulation 2006;114:e257-e354

2. Rahman F, Kwan GF, Benjamin EJ. Global epidemiology of atrial fibrillation. Nat Rev Cardiol 2014;11:639-654

3. De Sensi F, De Potter T, Cresti A, Severi S, Breithardt G. Atrial fibrillation in patients with diabetes: molecular mechanisms and therapeutic perspectives. Cardiovasc Diagn Ther 2015;5:364-373

4. Dublin S, Glazer NL, Smith NL, et al. Diabetes mellitus, glycemic control, and risk of atrial fibrillation. J Gen Intern Med 2010;25:853-858

5. Zhou J, Sun L, Chen L, Liu S, Zhong L, Cui M. Comprehensive metabolomic and proteomic analyses reveal candidate biomarkers and related metabolic networks in atrial fibrillation. Metabolomics 2019;15:96

6. Shah SH, Kraus WE, Newgard CB. Metabolomic profiling for the identification of novel biomarkers and mechanisms related to common cardiovascular diseases: form and function. Circulation 2012;126:1110-1120

7. Lee MY, Hu T. Computational methods for the discovery of metabolic markers of complex traits. Metabolites 2019;9:66

8. Tham YK, Bernardo BC, Huynh $\mathrm{K}$, et al. Lipidomic profiles of the heart and circulation in response to exercise versus cardiac pathology: a resource of potential biomarkers and drug targets. Cell Rep 2018;24:2757-2772

9. Patel A, MacMahon S, Chalmers J, et al.; ADVANCE Collaborative Group. Effects of a fixed combination of perindopril and indapamide on macrovascular and microvascular outcomes in patients with type 2 diabetes mellitus (the ADVANCE trial): a randomised controlled trial. Lancet 2007; 370:829-840

10. Alshehry ZH, Mundra PA, Barlow CK, et al. Plasma lipidomic profiles improve on traditional risk factors for the prediction of cardiovascular events in type 2 diabetes mellitus. Circulation 2016;134:1637-1650

11. Du X, Ninomiya T, de Galan B, et al.; ADVANCE Collaborative Group. Risks of cardiovascular events and effects of routine blood pressure lowering among patients with type 2 diabetes and atrial fibrillation: results of the ADVANCE study. Eur Heart J 2009;30:1128-1135

12. Shioi T, Kang PM, Douglas PS, et al. The conserved phosphoinositide 3-kinase pathway determines heart size in mice. EMBO J 2000;19:25372548

13. Weir JM, Wong G, Barlow CK, et al. Plasma lipid profiling in a large populationbased cohort. J Lipid Res 2013;54:2898-2908

14. Alonso A, Krijthe BP, Aspelund T, et al. Simple risk model predicts incidence of atrial fibrillation in a racially and geographically diverse population: the CHARGE-AF consortium. J Am Heart Assoc 2013;2:e000102

15. Hsueh W, Abel ED, Breslow JL, et al. Recipes for creating animal models of diabetic cardiovascular disease. Circ Res 2007;100:1415-1427

16. McMullen JR, Shioi T, Zhang L, et al. Phosphoinositide 3-kinase $(\mathrm{p} 110 \alpha)$ plays a critical role for the induction of physiological, but not pathological, cardiac hypertrophy. Proc Natl Acad Sci U S A 2003;100:12355-12360

17. McMullen JR, Amirahmadi F, Woodcock EA, et al. Protective effects of exercise and phosphoinositide 3-kinase $(\mathrm{p} 110 \alpha)$ signaling in dilated and hypertrophic cardiomyopathy. Proc Natl Acad Sci U S A 2007;104:612-617

18. Pretorius $L$, Du XJ, Woodcock EA, et al. Reduced phosphoinositide 3-kinase (p110 $\alpha$ ) activation increases the susceptibility to atrial fibrillation. Am J Pathol 2009; 175:998-1009

19. Sapra G, Tham YK, Cemerlang N, et al. The small-molecule BGP-15 protects against heart failure and atrial fibrillation in mice. Nat Commun 2014; 5:5705

20. Jung $\mathrm{Y}, \mathrm{Cho} \mathrm{Y}, \mathrm{Kim} \mathrm{N}$, et al. Lipidomic profiling reveals free fatty acid alterations in plasma from patients with atrial fibrillation. PLoS One 2018;13:e0196709

21. Del Greco M F, Foco L, Teumer A, et al. Lipidomics, atrial conduction, and body mass index. Circ Genom Precis Med 2019;12:e002384

22. Alonso A, Yin X, Roetker NS, et al. Blood lipids and the incidence of atrial fibrillation: the Multi-Ethnic Study of Atherosclerosis and the Framingham Heart Study. J Am Heart Assoc 2014;3:e001211

23. Li X, Gao L, Wang Z, et al. Lipid profile and incidence of atrial fibrillation: a prospective cohort study in China. Clin Cardiol 2018;41:314-320

24. Liu C, Geng J, Ye X, et al. Change in lipid profile and risk of new-onset atrial fibrillation in patients with chronic heart failure: a 3-year follow-up observational study in a large Chinese hospital. Medicine (Baltimore) 2018;97:e12485

25. Inokuchi J. Physiopathological function of hematoside (GM3 ganglioside). Proc Jpn Acad Ser B Phys Biol Sci 2011;87:179-198 


\section{University Library}

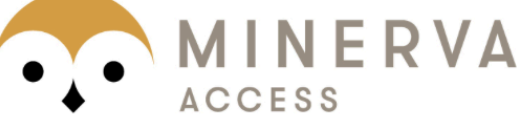

A gateway to Melbourne's research publications

Minerva Access is the Institutional Repository of The University of Melbourne

Author/s:

Tham, YK;Jayawardana, KS;Alshehry, ZH;Giles, C;Huynh, K;Smith, AAT;Ooi, JYY;Zoungas, S;Hillis, GS;Chalmers, J;Meikle, PJ;McMullen, JR

Title:

Novel Lipid Species for Detecting and Predicting Atrial Fibrillation in Patients With Type 2 Diabetes

Date:

2021-01-01

Citation:

Tham, Y. K., Jayawardana, K. S., Alshehry, Z. H., Giles, C., Huynh, K., Smith, A. A. T., Ooi, J. Y. Y., Zoungas, S., Hillis, G. S., Chalmers, J., Meikle, P. J. \& McMullen, J. R. (2021).

Novel Lipid Species for Detecting and Predicting Atrial Fibrillation in Patients With Type 2 Diabetes. DIABETES, 70 (1), pp.255-261. https://doi.org/10.2337/db20-0653.

Persistent Link:

http://hdl.handle.net/11343/277000 